\title{
WI - Call for Papers Heft 5/2015
}

\section{Economics and Value of IS}

DOI 10.1007/s11576-014-0418-2

\section{Die Autoren}

Assoc. Prof. Kunsoo Han, Ph.D.

McGill University Montreal

Montreal

Kanada

kunsoo.han@mcgill.ca

\section{Prof. Dr. Dennis Kundisch \\ Universität Paderborn \\ Paderborn \\ Deutschland \\ dennis.kundisch@ \\ wiwi.uni-paderborn.de}

Prof. Dr. Christof Weinhardt

Karlsruhe Institute of Technology

Karlsruhe

Deutschland

weinhardt@kit.edu

\section{Ass.-Prof. Dr. Steffen \\ Zimmermann (『) \\ Universität Innsbruck \\ Innsbruck \\ Österreich \\ steffen.zimmermann@uibk.ac.at}

Online publiziert: 2014-04-17

This article is also available in English via http://www.springerlink.com and http://www.bise-journal.org: Han K, Kundisch D, Weinhardt C, Zimmermann S (2014) BISE - Call for Papers Issue 5/2015. Economics and Value of IS. Bus Inf Syst Eng. doi: 10.1007/s12599-014-0327-3.

(C) Springer Fachmedien Wiesbaden 2014

\section{Schwerpunktthema}

Ein Großteil unserer Ökonomie stützt sich auf die Wertschaffung durch Informationssysteme (IS), welche die Gewinnung, den Austausch und die Ver- arbeitung großer Informationsmengen ermöglichen. Hieraus erwachsen einerseits neue Geschäftsmodelle, wie die an Bedeutung gewinnende „Sharing Economy“, und anderseits ergeben sich neue Möglichkeiten für Unternehmen, wie kundenindividuelle Produktanpassungen, die Analyse von Kunden erzeugter Inhalte oder die Kommunikation und den Vertrieb über soziale Netzwerke. Allerdings stehen noch immer fundierte Untersuchungen aus, wie sich diese neuen Geschäftsmodelle und Möglichkeiten auf Unternehmen, Industrien, Ökonomien und Gesellschaften langfristig auswirken. Das Schwerpunktheft zum Thema "Economics and Value of IS“ nimmt dies zum Anlass und behandelt dazu verschiedene IS- und IT-relevante Kernfragestellungen einschließlich der Themen Ökonomie von Informationsgütern, CloudComputing, mobile Dienstleistungen sowie den Einfluss von IT-getriebenen Geschäftsmodellen auf bestehende Organisationen, Industrien, Ökonomien und Gesellschaften.

Alle Einreichungen für das Schwerpunktheft sollten einen originären Theorie- oder Praxisbeitrag zur Gewinnung, Verarbeitung und Nutzung von IT liefern. Dies kann aus der Untersuchung neuer Phänomene, der Anwendung einer neuen theoretischen Perspektive oder dem Sammeln neuer Daten hervorgehen. Die Autoren sollten insbesondere ihren originären Erkenntnisfortschritt artikulieren und diesen vom Stand der Literatur abgrenzen.

Für das Schwerpunktheft können gestaltungsorientierte, theoretische und empirische Beiträge eingereicht werden. Interessante Beiträge adressieren - sind aber nicht beschränkt auf - folgende Themen:

- Ökonomie von Informations- und Kommunikationsgütern (Bepreisung, Güterbündel etc.)

- Geschäftsmodelle für Informationsgüter
- Ökonomie von Wertschöpfungsnetzwerken

- Internetwirtschaft

- Cloud-Computing

- Matching in elektronischen Märkten Fragen der Effizienz und des „Mechanism Designs“

- Ökonomie von mobile Dienstleistungen

- Entscheiden in elektronischen Märkten

- Netzwerkeffekte bei IS

- Ökonomie von IT Infrastrukturen

- Wertbeitrag von IT-Projekten und Programmen

- IT, Produktivität und organisationale Komplemente

- Online-Auktionen (u. a. Vertrauen, Simultanität und konkurrierende Auktionen)

- Plattformwettbewerb, -standards und mehrseitige Netzwerke

- Vorausschauende Modellierung in der IS

- Ökonomie des Teilens (Sharing Economy)

\section{Einreichung von Beiträgen}

Die Beiträge sind bis zum 2014-1101 online über das Einreichungssystem Editorial Manager (http://www. editorialmanager.com/buis/) unter der Kategorie „BISE - Economics and Value of IS" einzureichen. Für alle Beiträge gelten die Formatierungsvorgaben der WIRTSCHAFTSINFORMATIK/ Business \& Information Systems Engineering (BISE), die unter http://www. bise-journal.org einsehbar sind. Demnach dürfen Manuskripte höchstens 50.000 Zeichen enthalten (abzüglich 5.000 Zeichen für jedes Bild bzw. Tabelle). Detaillierte Richtlinien für Autoren können unter http://www.bise-journal.org bezogen werden. 
Es werden ausschließlich englische Einreichungen berücksichtigt. Alle Beiträge werden in einem doppelt blinden Begutachtungsverfahren hinsichtlich ihrer Relevanz, Originalität und fachlichen Qualität beurteilt. Neben den Herausgebern des Schwerpunktheftes und jenen der Zeitschrift wirken dabei weitere ausge- wiesene internationale Persönlichkeiten aus Wissenschaft und Praxis mit.

\section{Zeitplan}

Einreichung von Beiträgen: 2014-11-01 Benachrichtigung der Autoren: 2015-01-10
Abschluss der ersten Überarbeitungsphase: 2015-02-28

Benachrichtigung der Autoren:

2015-04-18

Abschluss der zweiten Überarbeitungsphase: 2015-05-23

Geplanter Veröffentlichungszeitpunkt: Oktober 2015 